\title{
Synergistic Smart Morphing Aileron: Capabilities Identification
}

\author{
Alexander M. Pankonien ${ }^{1}$ and Lawren L. Gamble ${ }^{2}$ \\ Department of Aerospace Engineering, University of Michigan, Ann Arbor, Michigan, 48109, USA \\ Cassio T. Faria ${ }^{3}$ \\ Siemens Industry Software NV, Leuven, Belgium \\ and \\ Daniel J. Inman ${ }^{4}$ \\ Department of Aerospace Engineering, University of Michigan, Ann Arbor, Michigan, 48109, USA
}

\begin{abstract}
Unmanned Aerial Vehicles (UAVs) can require adaptation to large changes in flight condition but are restricted by their size. Hybrid actuation systems that excel at both long timescale trim conditions and short timescale deviations, e.g. gusts, can be utilized to augment UAVs controllability. Previously, the Synergistic Smart Morphing Aileron (SSMA) system, a camber-morphing airfoil system utilizing two smart-material actuators operating over different timescales, was introduced and characterized without positional controllers. The combined SSMA system utilizes the comparatively high blocking force of shape-memory alloy (SMA) wires to augment static displacement under high aerodynamic loads while maintaining high-bandwidth tip displacement from the faster piezo-driven MFC actuator. Static aeroelastic simulations have shown that the concept can achieve superior control over flow separation through reflex actuation under aerodynamic loading. This current work further identifies performance capabilities of the SSMA concept that are synergistic in nature, and thus extended beyond that of the constituent actuators in isolation. Dynamic URANS simulations show that allowing the comparatively faster MFC-driven actuator to compensate for the slower SMA wires by actuating through reflex camber mimics the forces of a faster monotonic actuator. By implementing position controllers, an experimental demonstrator is used to initially validate both the static and dynamic benefits of synergistic force control through a hybrid morphing system. Although specific to this configuration, this study exposes how a multi-material, multi-timescale morphing concept can be leveraged to improve steady and unsteady flow control.
\end{abstract}

\section{Nomenclature}

$\alpha \quad=$ angle of attack

$C_{D}=$ drag force coefficient

$C_{L}=$ lift force coefficient

$C_{M}=$ pitching moment coefficient

$C_{P}=$ pressure coefficient

$M F C=$ Macro Fiber Composite

$M F C_{\delta}=$ Contribution of MFC-driven actuator to trailing edge deflection

$S M A=$ Shape Memory Alloy

$S M A_{\delta}=$ Contribution of SMA-driven actuator to trailing edge deflection

\footnotetext{
${ }^{1}$ Post-Doctoral Researcher, Department of Aerospace Engineering, 1320 Beal Avenue, AIAA Member.

${ }^{2} \mathrm{Ph} . \mathrm{D}$ Candidate, Department of Aerospace Engineering, 1320 Beal Avenue, AIAA Member.

${ }^{3}$ Researcher, Engineering Service, Siemens Industry Software NV, Leuven, Belgium, AIAA Member.

${ }^{4}$ Collegiate Professor and Chair, Department of Aerospace Engineering, 1320 Beal Avenue, AIAA Fellow.
} 


\section{Introduction}

$\mathrm{H}$ YBRID smart-material morphing airfoil concepts seek to bridge the deficiencies between singular morphing concepts by leveraging the advantages of their constitutive materials. In the process, new capabilities can be realized that were not previously achievable via the individual actuation concepts. However, to adequately leverage these capabilities, an accurate understanding of both the individual actuation concepts and the system as a whole is required. This barrier has previously prevented hybrid smart material morphing concepts from additional investigation.

On the scale of regional-transport aircraft, the Boeing/NASA Variable Camber Continuous Trailing Edge Flap (VCCTEF) has modeled the static aerodynamic performance of a segmented morphing wing concept with multiple time-scale actuators ${ }^{1}$. The concept utilized Shape Memory Alloy (SMA) torque tubes to alter long time-scale trim aerodynamics while electromechanical actuators control short time-scale flight control. Although not a multiple smart material concept, the concept illustrated the basic principle of hybrid morphing, i.e. utilizing different actuators to achieve complex configurations and operation over different timescales. Since its initial concept development, in addition to other characterization and modeling, the design has been statically tested on a subsonic scale for validation of modeled aeroelastic performance ${ }^{2}$.

On the scale of a UAV wing, Chinaud et al. sought to leverage the different actuation timescales for smart materials and piezoelectrics on a trailing edge aileron for a UAV wing ${ }^{3}$. Initially realized as a flat plate with a segmented foreward section driven by Shape Memory Alloy wires and a rigid trailing edge driven by piezoelectric stack actuators, the concept sought to control different timescale vortices through periodic high-frequency actuation. A later iteration of this concept utilized a conventional, thick, NACA airfoil with a compliant trailing edge. Timedependent PIV was utilized to investigate the power spectral density of trailing edge vortices near stall for both the flat plate ${ }^{4}$ and the thick NACA airfoil ${ }^{5}$.

The Synergistic Smart Morphing Aileron (SSMA) concept, which was developed independently, also utilized multiple smart material actuators for a UAV concept, but sought to exploit the quasi-static and transient nature of multiple actuators rather than high-frequency periodic actuation ${ }^{6}$. The SSMA represented a notional concept of a control surface on the scale of a Medium Altitude Long-Endurance (MALE) UAV and leveraged two smartmaterials as actuators, a piezoelectrically-driven Macro-Fiber Composite (MFC) and phase-transformation driven SMAs. The physical realization of these actuation mechanisms were a SMA-driven hinge and MFC-driven Flexure Box aileron.

As implemented in a representative configuration shown in Figure 1, the profile of a NACA 0012 airfoil with a $305 \mathrm{~mm}$ chord is augmented with two antagonistic pre-strained SMA wires (shown in blue) which rotate a discrete hinge at $160 \mathrm{~mm}$ chord and an elastic trailing edge deformable in bending via two antagonistic MFC unimorphs (shown in red). In this concept, the elastic trailing edge is generally noted as the "Flexure Box" . A derivative of the compliant hinged-box concept first developed by Bilgen et al. ${ }^{8}$, the trailing edge implemented in this work utilizes an internal elastomeric flexure mechanism capable of high precision positioning free of frictional losses. The serieslike configuration of the two actuators allows for reflex as well as monotonic actuation. Initial work by Pankonien $e t$ al. performed spectral characterization of the trailing edge deflection, without investigation of the resultant aerodynamic forces. Furthermore, initial efforts in modeling the force performance of the elastic structure under static aerodynamic loads by Pankonien et al. ${ }^{9}$ identified that reflex actuation, as shown in Figure 2, can be useful in unloading the internal compliant mechanism while reducing drag near stall.

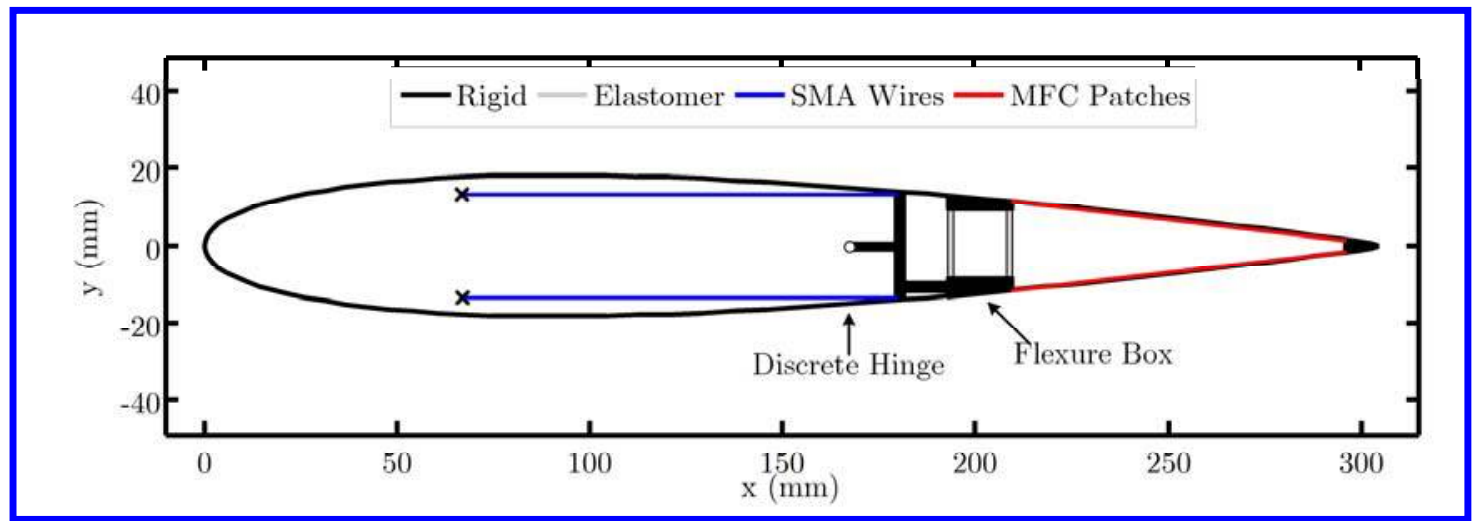

Figure 1. Diagram of Synergistic Smart Morphing Aileron Concept 

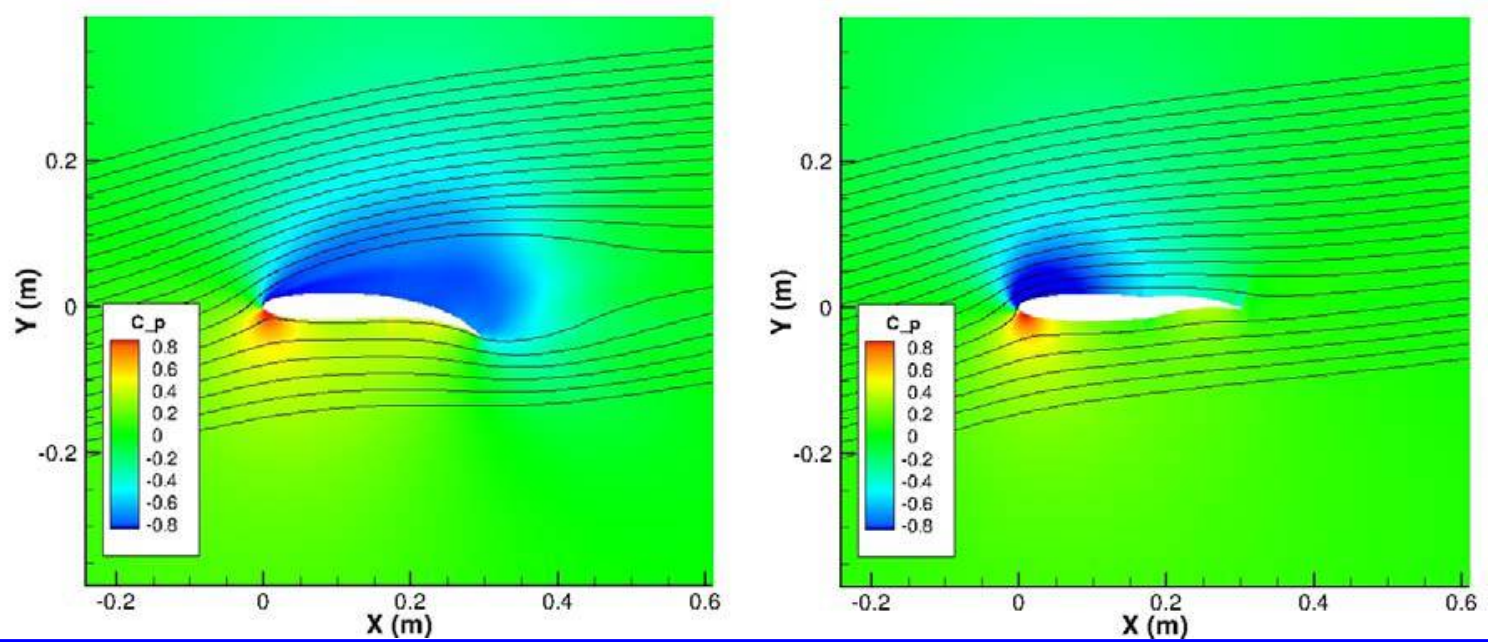

Figure 2. Monotonic vs reflex actuation of the SSMA for improved flow control of separation near stall ${ }^{9}$

\section{A. Scope of investigation}

The present work seeks not only to experimentally validate the previously-modeled static advantage of reflex actuation in a hybrid morphing concept, but to also investigate the benefit of dynamically actuating through reflex camber. The first section motivates the investigation of the dynamic benefits of reflex actuation through the introduction of the idea of a synergistic controller. In the second section, the expected performance of this controller on aerodynamic forces is simulated via Unsteady Reynolds Averaged Navier-Stokes simulations. Lastly, the third section experimentally validates both the expected static and dynamic aerodynamic force performance gains via a demonstrator.

\section{Description of Synergistic Controller}

Previous analysis of the trailing edge deflections of the SSMA concept showed that the primary quasi-static gains of the SSMA concept occurred between $0.1-1 \mathrm{~Hz}$, which corresponded to the gap between the rise in the relative time constant of the trailing edge deflection for the constituent actuators ${ }^{10}$. Although this result implied that the aerodynamic gains could also be augmented within this frequency range, the modeled first-order actuation initially resembled a step-response. At low flow speeds, relevant to a UAV, this sudden impulse could result in highly unsteady aerodynamic effects. Accordingly, it was desired to determine if these first-order tip-deflections would result in similar responses of aerodynamic control forces.

The purpose of the following sections was to then determine whether unsteady gains experimentally measured by the quasi-static characterization method for trailing edge deflections would also extend to aerodynamic forces such as lift, pitching moment, and drag for a representative, attached flow condition. The following section identifies a simplistic controller defined by the total trailing edge deflection of the hybrid morphing concept that will approximate the tip deflections of a monotonic actuator with a greater tip displacement range operating on a timescale similar to that of the faster subsystem.

\section{A. Simplified system response}

Previous work focused on experimentally determining a method to characterize the first-order response of the tip deflection of the hybrid SSMA concept and its constituent actuators to a step-like input response. The results concluded that both smart material systems could be initially characterized by measuring the response to a square wave input of a relevant period. For initial investigation, the effect of both smart material subsystems on the deflection of the trailing edge was assumed to be a first order system with a step input response of infinite period, of the form: 


$$
\frac{d \delta}{d t}+\frac{1}{\tau_{\infty}} \delta=f(t) \quad \text { where } \quad f(t)=\left\{\begin{array}{l}
\delta_{i} \text { for } t<0 \\
\delta_{f} \text { for } t \geq 0
\end{array}\right.
$$

where $\delta$ was the trailing edge displacement of the tip of the morphing aileron if everything else was held constant, $\delta_{i}$ the initial trailing edge position of the morphing aileron, $\delta_{f}$ was the final (desired) displacement, and $\tau_{\infty}$ was the firstorder time constant of the system response from Pankonien et al. ${ }^{10}$. Assuming that both of the initial and final positions were within the upper, $\delta_{u}$, and lower, $\delta_{l}$, bounds of the actuator, the response of the form:

$$
\Delta(t) \sim\left(1-e^{-t / \tau_{\infty}}\right)
$$

where $\Delta$ was the difference between the current position and the respective bound of the actuator in the direction of the desired actuation. For simplistic example, it was assumed that the actuator was already at the lower bound of the actuation, consistent with the characterization method from Pankonien et al. ${ }^{10}$. The initial and lower bounds of actuation were identical and the displacement can be written as:

$$
\delta(t)=\left(\delta_{u}-\delta_{l}\right)\left(1-e^{-t / \tau_{\infty}}\right)+\delta_{l} \quad \text { for } \quad \delta(t)<\delta_{f}
$$

where the system converged towards the opposite saturated condition until the desired condition was reached. Although this model ignored higher-order dynamic effects associated with sudden starting and stopping conditions, it assumed that the model would generally represent the expected path of an optimal control considering the firstorder limitations of the system.

Then the difference between the upper and lower bound of the respective actuators, denoted here by the name of the smart material driving the actuation system, were defined by:

$$
\begin{aligned}
& \Delta_{S M A}=\left(\delta_{u}-\delta_{l}\right)_{S M A} \\
& \Delta_{M F C}=\left(\delta_{u}-\delta_{l}\right)_{M F C}
\end{aligned}
$$

to generalize the modeling for the both the MFC-driven and SMA-driven actuators, respectively. As a result, the combined model system was represented as the summation of two first-order systems, characterized by the individual sub-system's time constants:

$$
\delta_{S S M A}(t)=\Delta_{M F C}\left(1-e^{-t / \tau_{M F C}}\right)+\Delta_{S M A}\left(1-e^{-t / \tau_{M F C}}\right)+\left(\delta_{l_{M F C}}+\delta_{l_{S M A}}\right)
$$

where $\delta_{\text {SSMA }}$ represented the trailing edge deflection of the combined, hybrid system.

\section{B. Hybrid synergistic control scheme}

Utilizing this simplified first-order system response, three different actuation configurations were investigated, representing both constituent actuator systems and the combined hybrid system. These configurations were abbreviated, similar to the previous description of the tip deflections, as: MFC, SMA and the SSMA. The SSMA configuration utilized both smart material actuation concepts. The MFC concept did not utilize the SMA concept and considered the actuation about the discrete hinge to be locked, or rigid. The SMA concept considered the trailing edge to be unactuated and rigid except for rotation about the discrete hinge.

Then, Equation (5) was simply amended to eliminate the terms for the inactive actuators, where the response for the SMA was: 


$$
\delta_{S M A}(t)=\Delta_{S M A}\left(1-e^{-t / \tau_{M F C}}\right)+\delta_{l_{S M A}} \text { for } \quad\left|\delta_{S M A}(t)-\delta_{\text {desired }}\right|>0
$$

and the response for the MFC-only configuration was:

$$
\delta_{M F C}(t)=\Delta_{M F C}\left(1-e^{-t / \tau_{M F C}}\right)+\delta_{l_{M F C}} \text { for }\left|\delta_{M F C}(t)-\delta_{\text {desired }}\right|>0
$$

and after the desired trailing edge configuration had been reached, the actuators remained at the desired position. For the combined system, rather than simply prescribing the actuations by Equation (5), the knowledge of the shorter timescale of the MFC actuator i.e. $\tau_{\mathrm{MFC}}<<\tau_{\mathrm{SMA}}$ was incorporated into the controller. In this synergistic control scheme, the desired position from the MFC-driven actuator was driven by the current position of the SMA, i.e.:

$$
\begin{aligned}
& \delta_{S M A}(t)=\Delta_{S M A}\left(1-e^{-t / \tau_{M F C}}\right)+\delta_{l_{S M A}} \text { for }\left|\delta_{S M A}(t)-\delta_{\text {desired }}\right|>0 \\
& \delta_{M F C}(t)=\delta_{\text {desired }}-\delta_{S M A}(t)
\end{aligned}
$$

Because the two actuation schemes in the SSMA were operated in series over the chord of the airfoil, this controller non-monotonically alters the airfoil's camber attempting to reach the desired trailing edge configuration. Although somewhat crude, this scheme embodied the basic principle of the synergistic controller for the hybrid actuation system: namely, the actuator with the faster time-scale compensated for the actuator with the slower timescale. The effect of this non-monotonic camber configuration on aerodynamic forces was investigated in the following sections.

\section{Modeled Dynamic Performance of Synergistic Controller}

For this hybrid concept, the synergistic controller then drives the comparatively-faster MFC-based actuator to compensate for the comparatively slower response of the SMA-based actuator through non-monotonic actuation, temporarily entering a state of reflex camber. Accordingly, it was desired to determine how this synergistic control would impact aerodynamic control forces and pressure for a representative flight condition

\section{A. Representative implementation of synergistic controller}

To compare the hybrid combined system with its constituent actuators, a representative actuation scenario was assumed where the airfoil was desired to adapt from a maximum additional lift condition to a zero additional lift condition in the minimum possible time. This scenario was chosen to correspond with a typical UAV roll or pitch-up condition that was suddenly aborted. For clarity, an intuitive attached-flow scenario about $\alpha=0^{\circ}$ was selected. In this scenario, the positional configuration prescribing zero lift was already known, specifically a zero trailing edge deflection with no camber. Similarly, the maximum lift condition was the maximum trailing edge deflection downward. This scenario was investigated for three configurations: the combined hybrid system (or SSMA), the MFC-driven trailing edge acting alone (MFC-only), and the SMA-driven hinge acting alone (SMA-only) to better identify the effects of the various actuation systems

Utilizing the first-order time constants and actuator ranges from Pankonien et al. the trailing edge displacement was prescribed for the representative control scenario. The resultant trailing edge displacements and normalized actuation bounds of the various configurations summarized in Figure 3.

As seen in Figure 3a, the trailing edge deflection of the SSMA achieved the desired value in approximately 0.33 $\mathrm{s}$, which was slightly longer than the MFC actuating alone $(0.11 \mathrm{~s})$. This slower actuation was a result of the SSMA starting at a maximum downward tip deflection that was $60 \%$ larger than that of the MFC-only actuator due to the combination of series actuation. As a result, the MFC traveled further across its achievable range to compensate for the SMA, seen in the comparison of actuations presented in Figure 3b. Upon reaching the desired neutral total tip deflection, the MFC slowly varied its tip deflection in proportion to the SMA. The compensation of the MFC actuator within the SSMA ended after approximately 2.1 seconds when the SMA actuator finally reached its neutral un-cambered position. 


\section{B. Modeled results}

The airfoil profiles associated with the three configurations were converted into a set of meshes for transient analysis in a URANS code, namely OVERTURNS ${ }^{11}$. A representative flight condition of $\alpha=0^{\circ}$ and $\mathrm{V}_{\infty}=20 \mathrm{~m} / \mathrm{s}$ was chosen for evaluation and a sufficiently small time step of $1 / 270 \mathrm{~s}$ was used to iteratively evaluate the unsteady forces due to actuation after converging the initial steady condition. The resultant shapes and the influences of these shapes on the flow about the SSMA configuration were illustrated via several relevant pressure fields, plotted in Figure 4, to highlight the non-monotonic or dynamic "reflex" utilized by the synergistic controller and its resultant effect on the pressure distribution.
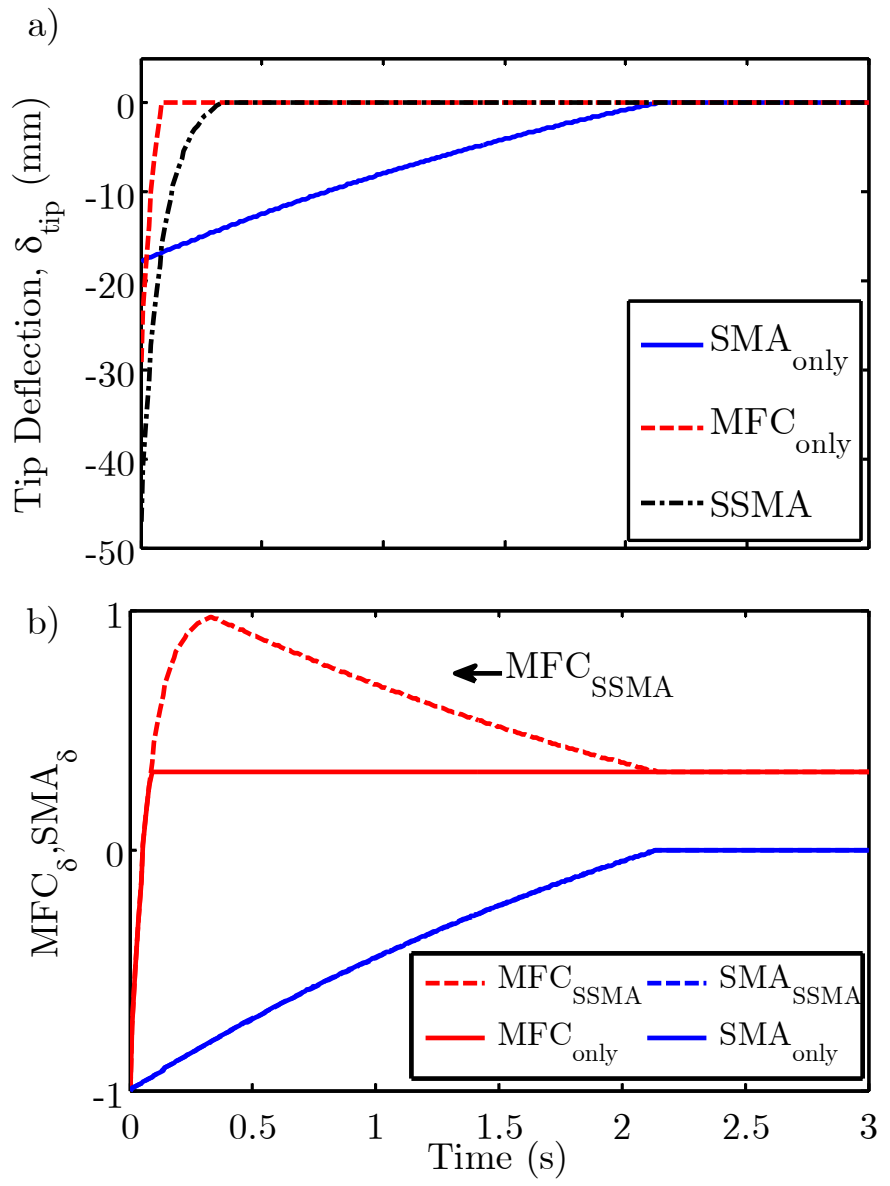

Figure 3. a) Prescribed tip deflection for SSMA as compared to constituent actuators and b) resultant actuation amounts for MFC and SMA in both cases 

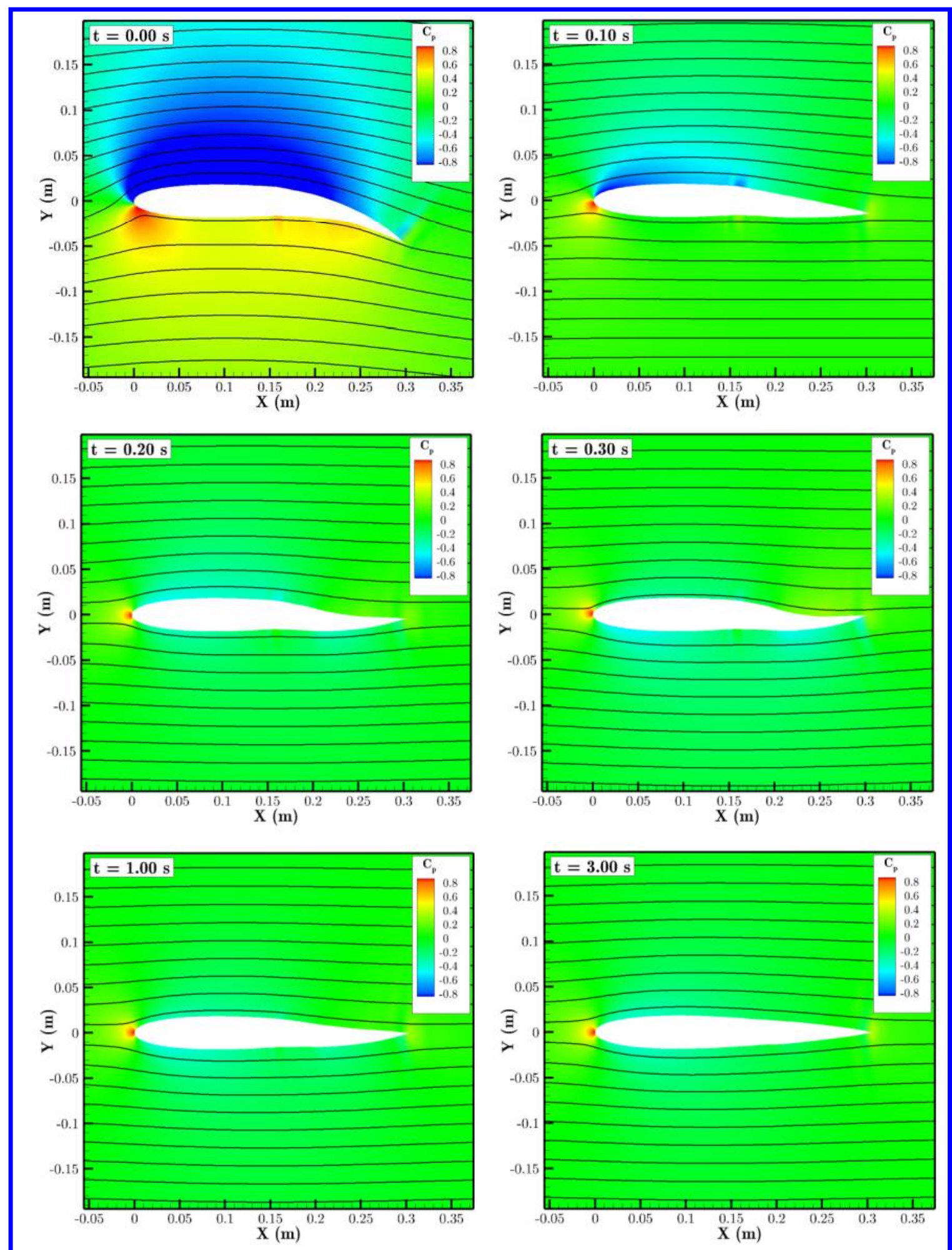

Figure 4. Unsteady pressure field for SSMA with synergistic controI for $V \propto=20 \mathrm{~m} / \mathrm{s}$ and $\alpha=0^{\circ}$ and incrementing time 
With cursory analysis, it is easily seen that the pressure field for the SSMA actuation, at $\mathrm{t}=0.2 \mathrm{~s}$ much more closely resembled the final configuration at $t=3.0 \mathrm{~s}$ rather than the initial pressure distribution at $\mathrm{t}=0 \mathrm{~s}$. The pressure distribution at $\mathrm{t}=0.3$ represented one of the maximum reflex camber states, where a negative pressure distribution was found on the bottom of the airfoil, corresponding to a total negative lift, as reflected by the nondimensional lift force. Between $\mathrm{t}=0.3 \mathrm{~s}$ and $\mathrm{t}=3.0 \mathrm{~s}$, the reflex camber slowly relaxed until the SMA actuator had reached its neutral position, and the symmetric flow had been restored, approaching form below.

The results of the nondimensional aerodynamic forces measured about the quarter chord of the wing were shown in Figure 5a and a shorter timescale of the forces prior to 0.5 seconds shown in Figure $5 \mathrm{~b}$ over the maximum compensation by the MFC-based actuator in the SSMA concept. As seen in Figure 5a, the reflex actuation resulted in negative lift as the SSMA overshot the zero-lift condition. However, by overshooting the desired lift condition, the SSMA reached zero-lift after approximately 0.2 seconds, which was almost $50 \%$ faster than when the trailing edge deflection first reached zero. The reflex configuration also resulted in a pitching moment offset that persisted until the SMA had reached its desired position. Also, the drag of the SSMA reached zero during the early phases of reflex actuation.

From these initial results, reflex camber was further motivated as a novel unsteady technique that could not only reproduce trailing edge deflections approximating a larger, fast actuator, but also alter the aerodynamic control forces on the timescale of the faster actuation system.
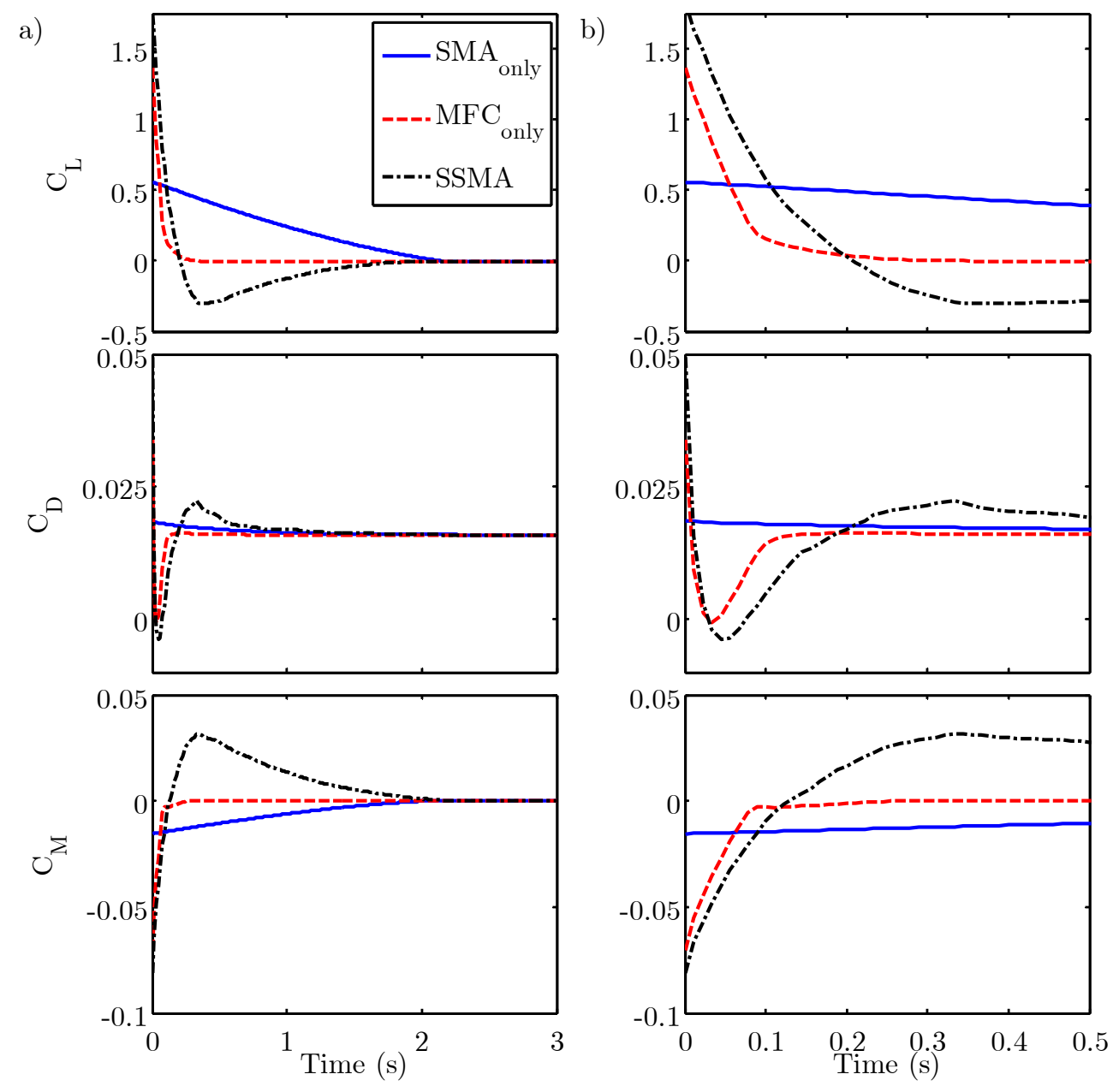

Figure 5. a) Unsteady aerodynamic forces for SSMA compared to constituent actuators and b) shortened time-scale view of aerodynamic forces 


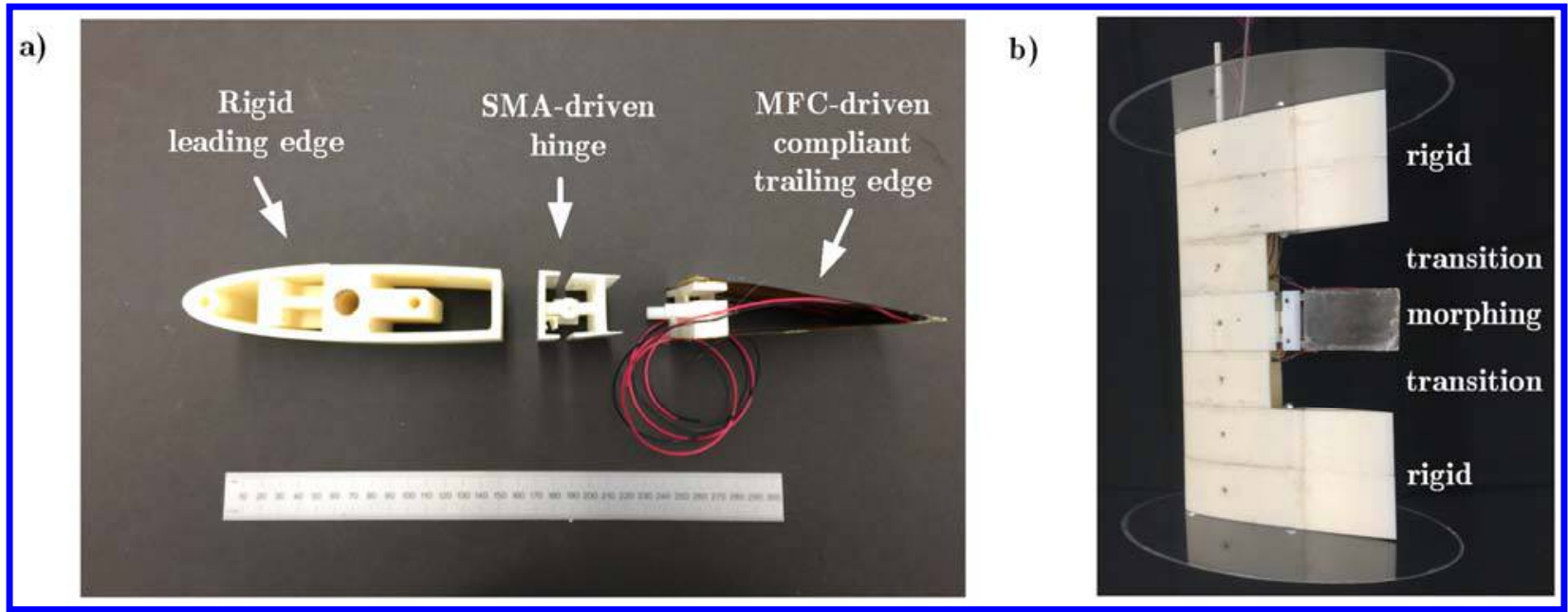

Figure 6. a) Deconstruction of modular SSMA section as part of a b) larger wing section with end plates

\section{Experimental Investigation}

Drawing from the modeled static and dynamic advantages of reflex actuation for this hybrid morphing system, an experimental demonstrator was created and tested to validate the expected gains in aerodynamic force performance. The following section characterizes the construction of and results from an experimental wind tunnel demonstrator designed to realize these gains.

\section{A. Test article description}

The SSMA hybrid concept was implemented as a finite airfoil with aspect ratio of 0.25 , shown deconstructed in Figure 6a with SMA-wire driven hinge interior SMA wires and MFC trailing edge unimorphs shown labeled recreating the demonstrator design from Figure 1. The morphing airfoil was embedded in the center of the span of a finite wing of aspect ratio 1.75 with end plates, as shown in Figure 6, to assure that the flow over the morphing section was largely two-dimensional and to minimize the effects of the endplates, permitting investigation of the morphing concept at stall.

Two variations in the skin were created to allow for testing of both a rigid trailing edge and a continuous trailing edge with the hybrid actuator. In the first variation, the transition regions of the trailing edge neighboring the morphing trailing edge, shown in shown in Figure 6b, were spanned initially with a thin, aluminum skin to emulate a rigid trailing edge, shown in Figure 7a. In the second variation of the skin, a 3D-printed elastomeric honeycomb skin, as previously developed by Pankonien et al. ${ }^{7}$, and shown in Figure $7 \mathrm{~b}$ was implemented to form a continuous trailing edge that permitted actuation of both morphing concepts while eliminating spanwise discontinuities that could create spanwise flow vortices, disrupting near-stall investigation.

The two trailing edge stations neighboring the morphing section were removed to permit appropriate transition regions from the center morphing section to the rigid airfoils on either side. 


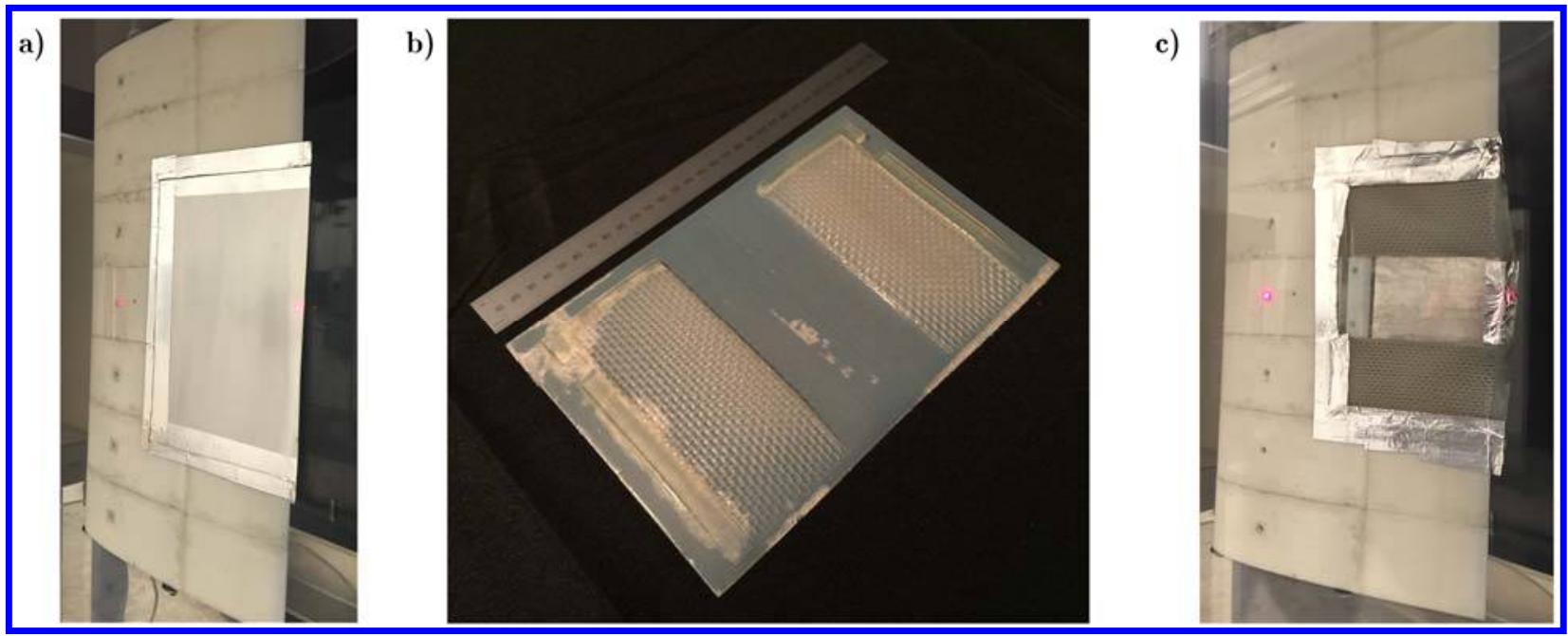

Figure 7. a) Rigid trailing edge configuration with thin aluminum skin spanning the transition regions B0 one panel of elastomeric honeycomb skin and c) morphing trailing edge with elastomeric honeycomb skin

The demonstrator was mounted cantilevered to a 6-axis force balance on the top of the wind tunnel to allow inplane measurement of the two-dimensional aerodynamic forces i.e. lift, drag, and pitching moment. The angle of attack was precisely prescribed utilizing a stepper motor accurate to $1 / 100^{\text {th }}$ of a degree. The dynamic pressure was monitored via a pitot pressure port mounted $0.4 \mathrm{~m}$ upstream of the test section that was connected to an electronic pressure transducer, which allowed for correction of dynamic pressure due to blockage via nondimensional force coefficients.

To implement both static positional control and the synergistic controller for the SSMA, analog sensors were used to provide calibrated, time-accurate assessments of the contribution of both actuators to the trailing edge deflection. A rotary potentiometer was placed in-line with the axis of the SMA-driven discrete hinge. Then, by using the potentiometer as a half-bridge Wheatstone circuit, the equivalent tip deflection was calibrated against the analog voltage output using a laser displacement sensor. In a similar manner to Pankonien et al. ${ }^{12}$ the flex sensors were added to the MFC-driven Flexure Box. Both sensing systems were calibrated independently with a laser displacement sensor located at the trailing edge of the morphing section.

The data acquisition was performed with a dSpace MicroLabBox running a compiled Simulink code, operating at $1 \mathrm{kHz}$. This device allowed for time-accurate controller and data acquisition within a single instrument. A closedloop controller was implemented for both actuators in the SSMA demonstrator described in Section II via closed loop control of the tip displacement via Simulink. The Flexure Box actuator was controlled utilizing a PI controller with representative gains set by the Ziegler-Nichols ${ }^{13}$ tuning method. The positioning error utilized in the MFConly controller was defined by the difference between the desired total tip deflection and the current total tip deflection. The SMA-driven hinge was controlled with an on-off directional controller that heated the desired wire at full power until the tip deflection of the SMA had reached the desired position within a prescribed error, $\varepsilon_{\mathrm{SMA}}$, which for initial testing, was set to $3 \mathrm{~mm}$, or $1 \%$ of the chord. Data was collected for varying flight conditions via a series of capture sequences that were setup, executed, and acquired via the XIL interface, which allowed for direct postprocessing of data in Matlab after each acquisition sequence.

\section{Static test}

Initially, static experiments were conducted to quantify the relevant regions linear, stall, and post-stall regions of the experimental setup and to identify areas of advantages in aerodynamic force coefficients through novel reflex actuation. 

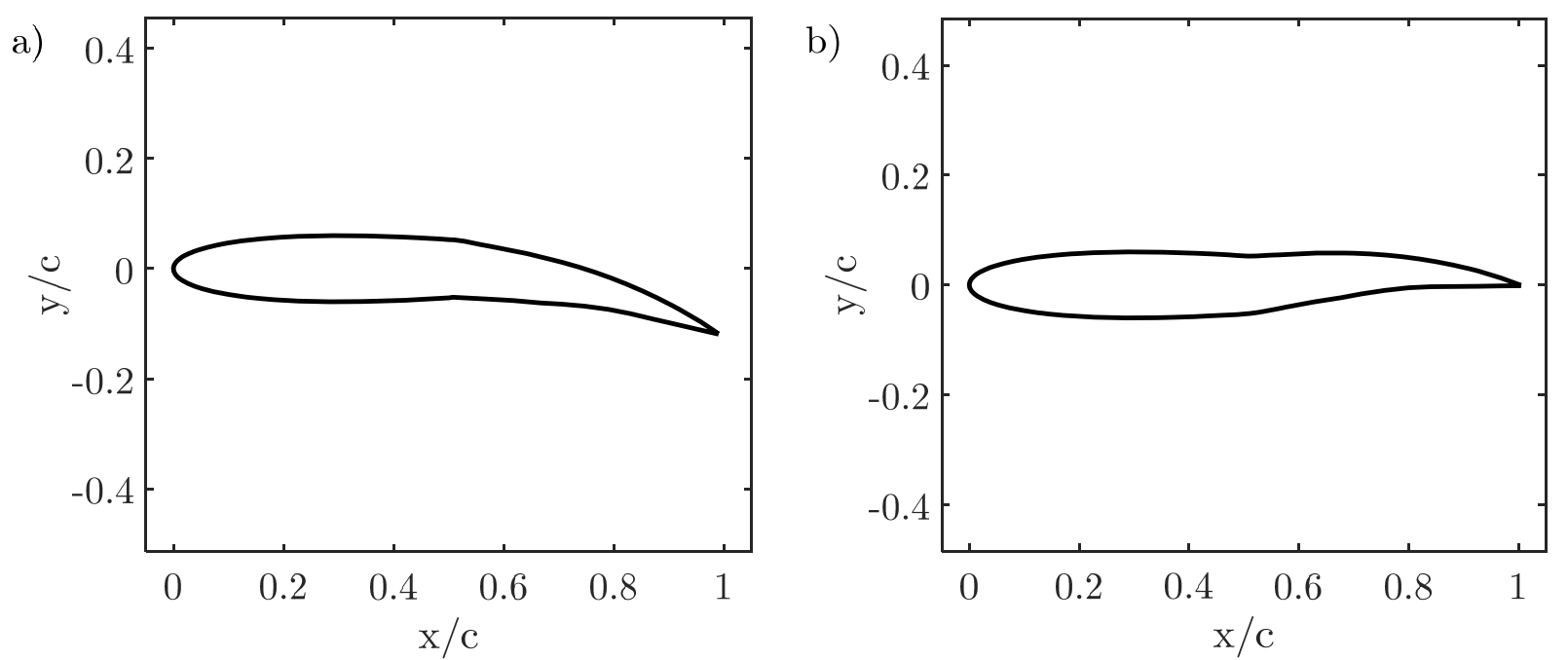

Figure 8. a) Monotonic actuation and b) reflex actuation

\section{Variation in angle of attack at constant actuations}

The first tested configuration, "Unactuated", was the rigid trailing edge, where the non-rigid portions of the SSMA were covered by the thin aluminum skin that was described in the previous section. This configuration served as an aeroelastic baseline that isolated the purely aerodynamic forces on the demonstrator. The second and third configurations utilized the 3D printed honeycomb skin to permit motion from the hybrid actuators.

The second tested configuration, "Monotonic Actuation", represented a monotonic actuation where both the SMA and MFC-driven actuators were actuated uniformly so that the overall trailing edge deflection was downward and the camber was monotonic creating positive lift. The specific case chosen was an overall trailing edge deflection of $2 \mathrm{~cm}$, where both actuation systems contributed $1 \mathrm{~cm}$, as shown in the diagram in Figure 8a, corresponding to a configuration of $\left[\mathrm{MFC}_{\delta}, \mathrm{SMA}_{\delta}\right]=[-1 \mathrm{~cm},-1 \mathrm{~cm}]$.

The third tested configuration, "Reflex Actuation", shown in Figure 8b, represented a non-monotonic actuation where the SMA and MFC-driven actuators were held in a reflex configuration so that the overall trailing edge deflection was zero, similar to the unactuated case, but the overall camber was non-zero and non-monotonic. The specific case chosen was an overall trialing edge deflection of $2 \mathrm{~cm}$, where the SMA-driven hinge was contributing $1 \mathrm{~cm}$ of equivalent trailing edge deflection upward, and the MFC-driven trailing edge was contributing $1 \mathrm{~cm}$ of equivalent trailing edge deflection downwards, corresponding to $\left[\mathrm{MFC}_{\delta}, \mathrm{SMA}_{\delta}\right]=[-1 \mathrm{~cm},+1 \mathrm{~cm}]$. This case was informed by the prior modeling results of Pankonien et al. ${ }^{9}$, and shown in Figure 2 that identified the possibility of reflex actuation improving control over flow separation near stall.

A representative flow speed of $10 \mathrm{~m} / \mathrm{s}$ was chosen and sweeps in angle of attack, $\alpha$, were performed for each configuration, recording the aerodynamic forces. Each data point was comprised of holding the respective configuration and angle of attack for 20 seconds, and time-averaging the forces. The resultant time-averaged forced were normalized by the span and chord of the demonstrator, as well as the dynamic flow pressure to calculate the aerodynamic force coefficients (i.e. $C_{L}, C_{D}$ and $C_{M}$ ).

Because the entire span of the demonstrator, rather than the span of the morphing section, was used for normalization, the reported values do not represent two-dimensional results for any of the actuated cases. Rather, the results were used to identify regions for potential flow control improvement via reflex actuation. Normalization via only the active span and direct comparison with modeled results was considered to be beyond the scope of the current investigation.

The nondimensional aerodynamic force results from the static test are shown in Figure 9. Identifying the various flow regimes, all configurations showed linear lift and pitch results until approximately $\alpha=10^{\circ}$. Stall occurred at approximately $\alpha=12^{\circ}$ as identified by the reduced slope in lift, and rapid drag rise. By $\alpha=15^{\circ}$, all configurations had stalled, resulting in drastically reduced lift to drag ratios. Interestingly, the lift generated by the reflex configuration began to exceed that of the other configurations around stall while operating at a lower drag coefficient. Accordingly, the reflex actuation configuration represented the highest lift-to-drag ratio configuration from approximately $\alpha=5^{\circ}$ to $12^{\circ}$ 

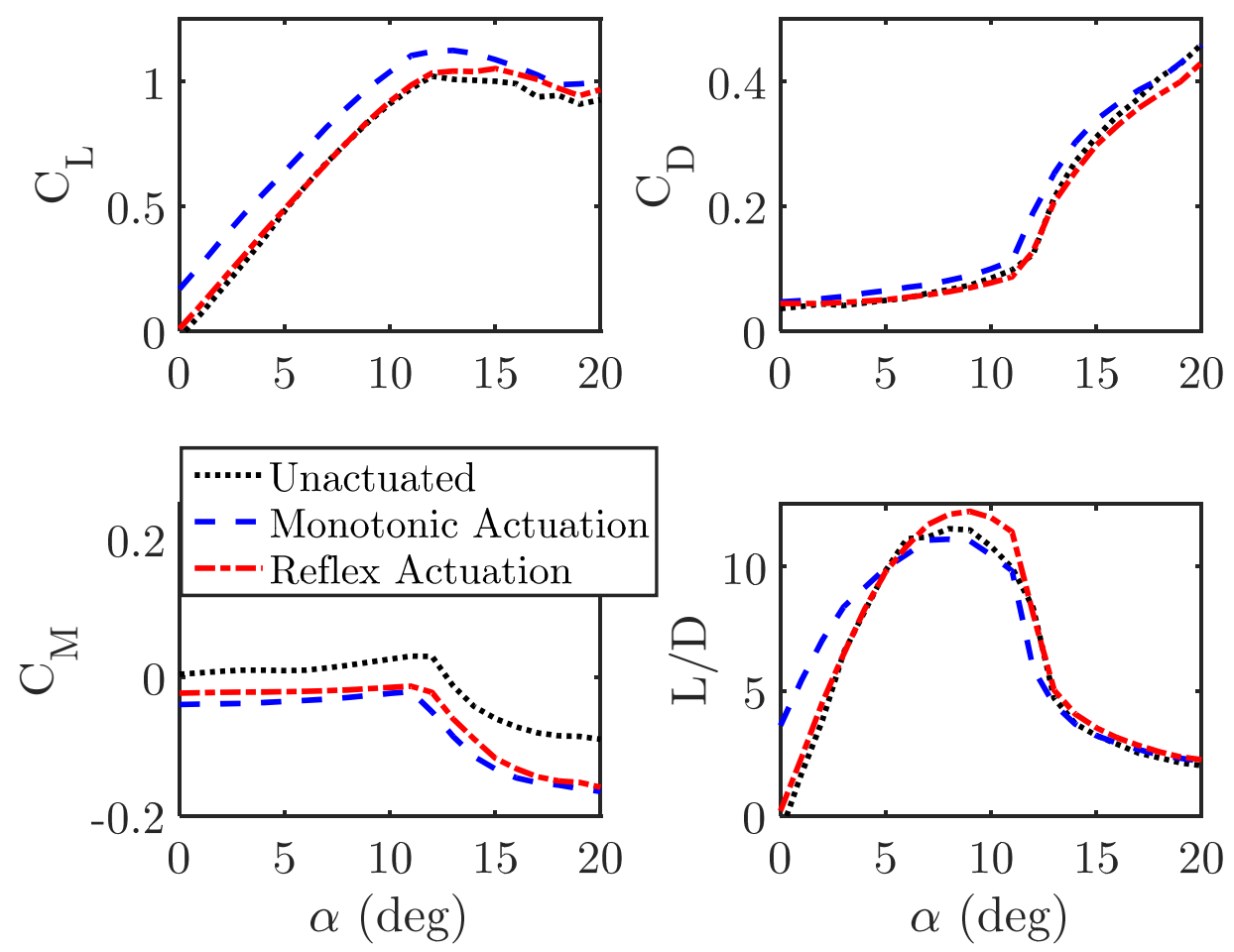

Figure 9. Nondimensional forces for variation in angle of attack with constant actuation

2. Variation in actuation at constant angle of attack

To compare the capabilities of monotonic and reflex actuation for the same flight condition, a single angle of attack was chosen to investigate variation in actuation capabilities. The stall angle of attack, $\alpha=12^{\circ}$, was chosen to highlight the ability of reflex actuation to control flow separation. While holding this angle of attack, varying amounts of monotonic and reflex actuation were prescribed.

The reflex configuration started at unactuated $[\mathrm{MFC}, \mathrm{SMA}]=[0 \mathrm{~cm}, 0 \mathrm{~cm}]$, and increased the reflex in equal increments until the prescribed deformation reached $[\mathrm{MFC}, \mathrm{SMA}]=[-2 \mathrm{~cm}, 2 \mathrm{~cm}]$. The monotonic configuration started at $[\mathrm{MFC}, \mathrm{SMA}]=[-2 \mathrm{~cm},-2 \mathrm{~cm}]$, and progressed in equal increments until $[\mathrm{MFC}, \mathrm{SMA}]=[2 \mathrm{~cm}, 2 \mathrm{~cm}]$, passing through the unactuated configuration, $[\mathrm{MFC}, \mathrm{SMA}]=[0 \mathrm{~cm}, 0 \mathrm{~cm}]$, midway through the test.

The lift-to-drag ratio for the two configurations was plotted against the lift coefficient as a surrogate for range performance at the same flight condition (i.e. matched angle of attack and lift condition), in Figure 10. For both monotonic and reflex actuation, the lift-to-drag ratio sharply increased as the flow un-stalled over the morphing portion of the demonstrator. The results clearly show a higher lift to drag ratio via reflex actuation over a range of matched geometric and force flight conditions. For example, at $\mathrm{C}_{\mathrm{L}}=1.08$ reflex actuation improved the lift to drag ratio by $16 \%$. These results highlight the capability of reflex actuation to decrease drag as compared to conventional monotonic morphing for the same flight condition near stall. 


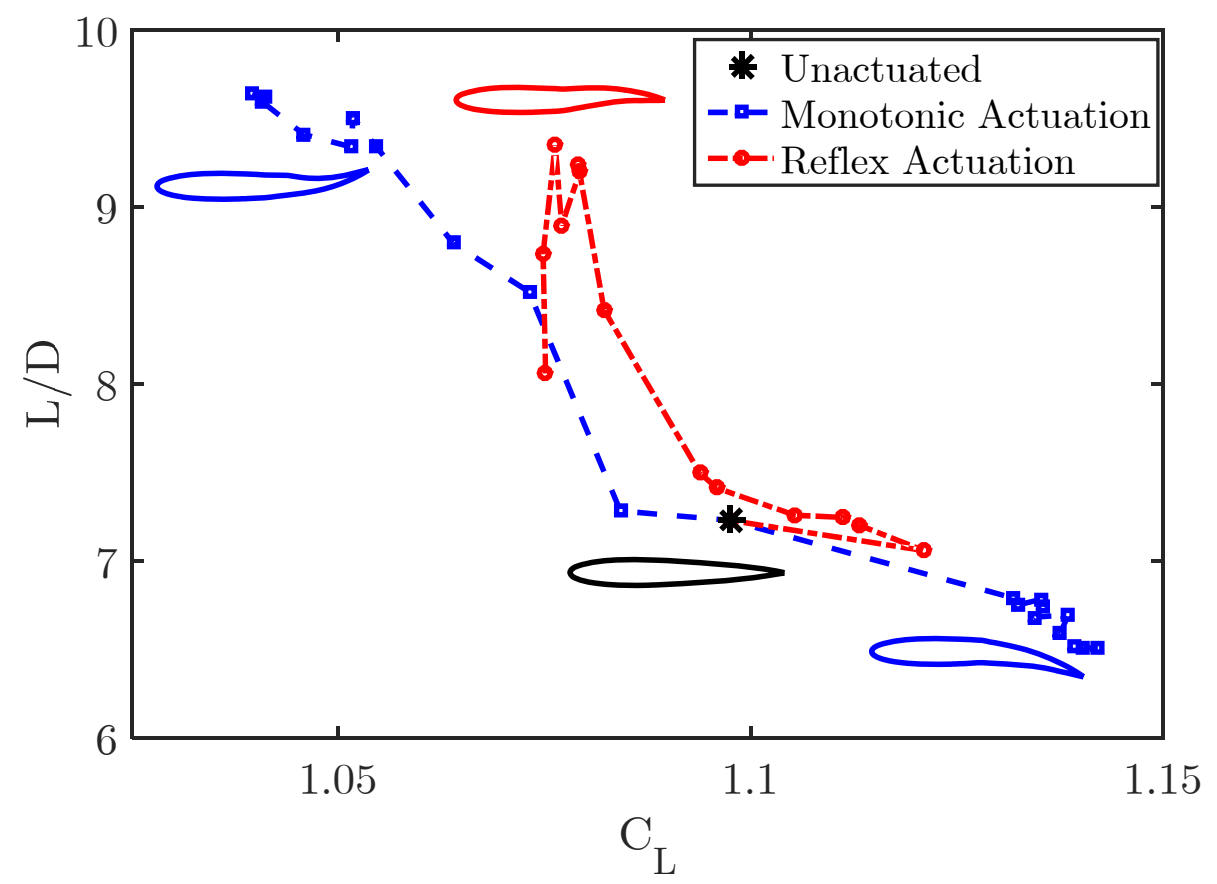

Figure 10. Lift to drag ratio vs lift coefficient for variation in actuation at stall $\left(\alpha=12^{\circ}\right)$

\section{Dynamic}

Similar to how the reflex configuration was used to extend the capabilities of the SSMA for stall in static cases, it was desired to determine if reflex actuation could provide the expected unsteady aerodynamic gains through use of the synergistic controller as predicted in Section III. For the previously modeled case, in the linear aerodynamic regime, reflex was shown to overshoot the predicted desired lift value, producing an actuator that reached the desired lift condition over a shorter timescale that would have been permitted by the longer-timescale actuator alone.

Thus, the previously-described synergistic controller was utilized, where the MFC positioning error in the Simulink controller was altered to included an additional term to compensate for the deviation of the SMA-driven actuator's current position from the total desired tip position. In a manner similar to Equation (8)

$$
\delta_{\text {desired }_{M F C}}(t)=\delta_{\text {desired }_{\text {total }}}-\delta_{\text {current }_{S M A}}(t)
$$

the desired positioning for the MFC $\delta_{\text {desired, } M F C}$, was defined by the difference between the total desired position $\delta_{\text {desired,total }}$ and the current equivalent tip position contribution by the SMA actuator, $\delta_{\text {current,SMA. }}$

\section{Dynamic actuated to unactuated for attached flow}

To reiterate the previous representative actuation case, it was defined by shifting from the maximum lift actuation case to no actuation through reflex actuation as quickly as possible. The flow speed was set for $10 \mathrm{~m} / \mathrm{s}$, angle of attack at $0^{\circ}$. 

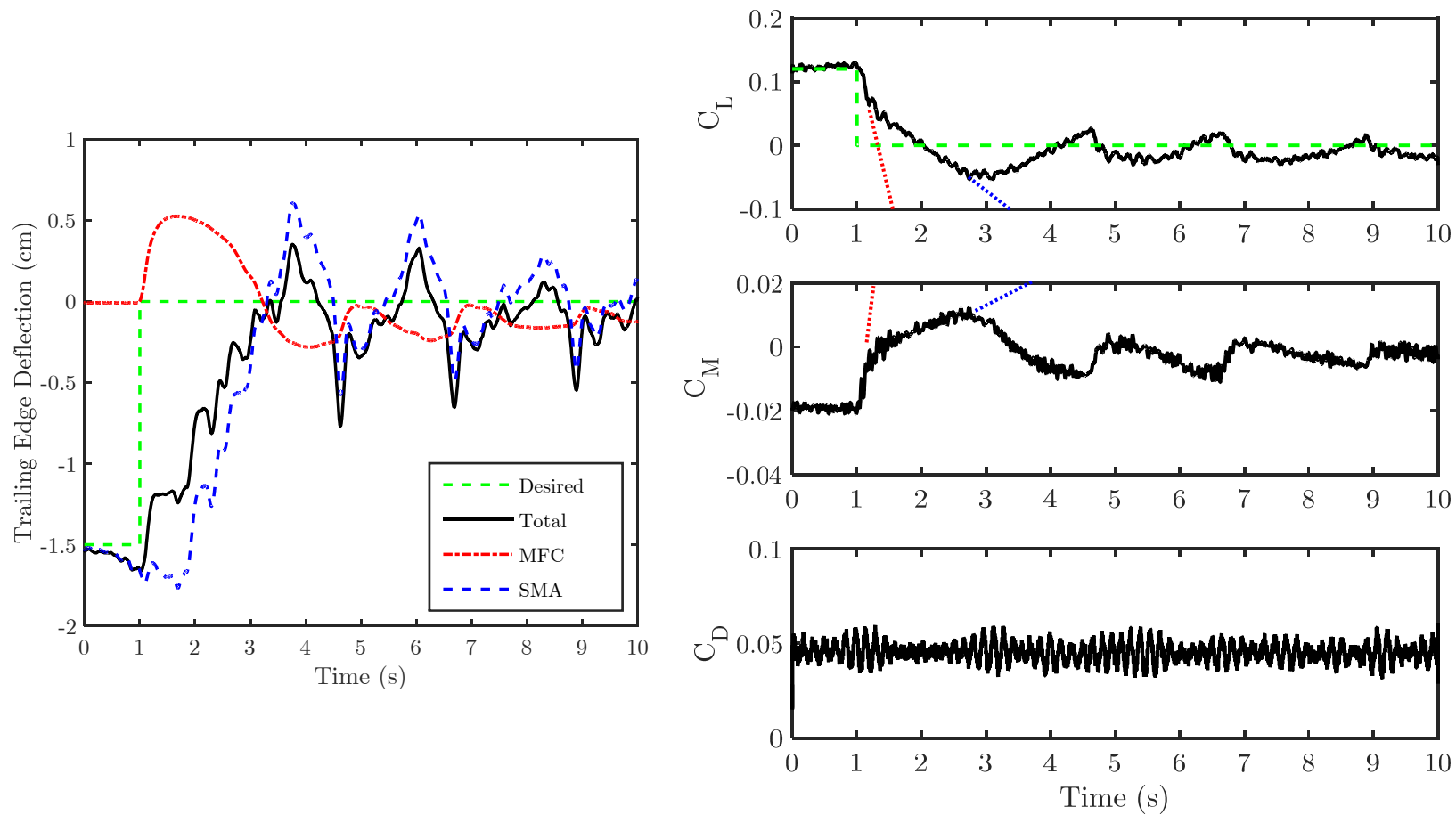

Figure 11. a) Convergence of tip displacement and b) nondimensional aerodynamic forces

The results from this representative case were shown in Figure 11. As shown by the step in desired trailing edge, the commanded change in position started at $\mathrm{t}=1 \mathrm{~s}$ into the sampling period. Over the first two seconds, until $\mathrm{t}=3.3 \mathrm{~s}$, the MFC-driven actuator actuated trailing-edge upward to cause the morphing section to enter into reflex until the SMA-driven actuator reached the unactuated position. However, the SSMA reached the zero lift condition at approximately $\mathrm{t}=2.02 \mathrm{~s}$, representing a $55 \%$ decrease in the total time to reach the zero-lift condition, utilizing the SMA actuator's displacement to characterize its independent convergence rate. The difference in slope between only the SMA-actuator converging to the desired location and both actuators converging to the desired force condition are shown in both the lift and pitching moment in Figure 11b. Due to the relatively low drag force near zero angle of attack, the drag coefficient only showed oscillations, most likely due to the structural modes of the demonstrator. Testing at higher angles of attack near stall could create larger drag forces that could be more detectable under dynamic actuation. Still, these results highlight the successful capability of the SSMA to leverage the faster actuator system and reflex actuation to approximate a larger monotonic actuator.

\section{Conclusions}

The SSMA, a hybrid multi-smart material morphing concept, was then shown to effectively exploit reflex actuation for both static aerodynamic force gains near stall and dynamic force gains via the use of a "synergistic" controller where the configuration of the faster trailing edge actuator was driven to compensate for the slower actuation system.

As implemented in a NACA 0012 demonstrator of $A R=1.75$ with $1 / 7$ th of its total span as active morphing airfoil and each neighboring $1 / 7$ th providing a transition region, static reflex actuation improved lift-to-drag ratio by $16 \%$ at stall $\left(\alpha=12^{\circ}\right)$, for a matched angle of attack and lift condition, confirming the modeled trend of improve flow control separation at stall through reflex actuation. As also modeled, dynamic reflex actuation could potentially result in unsteady aerodynamic benefits allowing the combined system to appear as a monotonic actuator with a larger range, acting over the timescale of the faster actuator. The dynamic experiments also showed the combined system converging over the faster timescale, and overshooting the desired lift condition. These results motivate additional investigation into the static and dynamic advantages of non-monotonic actuation via hybrid systems near stall, in conjunction with multi-point optimize design. 
Even though these advantages were for this specific hybrid configuration, the advantages and methods for achieving these advantages i.e. static reflex actuation, and a synergistic controller utilizing dynamic reflex actuation to approximate a larger monotonic actuator could be extended to other hybrid morphing concepts.

\section{Acknowledgements}

This work is supported in part by the US Air Force Office of Scientific Research under grant number FA 9550-16-1-0087, titled “Avian-Inspired Multifunctional Morphing Vehicles” monitored by Dr. BL Lee.

\section{References}

1 Ippolito, C., Nguyen, N., Totah, J., Trinh, K., and Ting, E., "Initial assessment of a Variable-Camber Continuous Trailing-Edge Flap system on a rigid wing for drag reduction in subsonic cruise," AIAA Infotech at Aerospace (I at A) Conference, 2013, DOI : 10.2514/6.2013-5143 .

-2 Precup, N., Mor, M., and Livne, E., "The design, construction, and tests of a concept aeroelastic wind tunnel model of a high-lift variable camber continuous trailing edge flap (HL-VCCTEF) wing configuration," 56th AIAA/ASCE/AHS/ASC Structures, Structural Dynamics, and Materials Conference, 2015, DOI : 10.2514/6.20151406 .

>3 Chinaud, M., Scheller, J., Rouchon, J., Duhayon, E., and Braza, M., "Hybrid electroactive wings morphing for aeronautic applications," Diffusion and Defect Data Pt.B: Solid State Phenomena, vol. 198, 2013, pp. 200-205, DOI : 10.4028/www.scientific.net/SSP.198.200 .

-4 Chinaud, M., Rouchon, J. F., Duhayon, E., Scheller, J., Cazin, S., Marchal, M., and Braza, M., "Trailing-edge dynamics and morphing of a deformable flat plate at high Reynolds number by time-resolved PIV," Journal of Fluids and Structures, vol. 47, 2014, pp. 41-54, DOI : 10.1016/j.jfluidstructs.2014.02.007 .

$\checkmark 5$ Scheller, J., Chinaud, M., Rouchon, J. F., Duhayon, E., Cazin, S., Marchal, M., and Braza, M., "Trailing-edge dynamics of a morphing NACA0012 aileron at high Reynolds number by high-speed PIV," Journal of Fluids and Structures, vol. 55, 2015, pp. 42-51, DOI : 10.1016/j.jfluidstructs.2014.12.012 .

$\checkmark 6$ Pankonien, A. M., Faria, C. T., and Inman, D. J., "Synergistic smart morphing aileron," 54th AIAA/ASME/ASCE/AHS/ASC Structures, Structural Dynamics, and Materials Conference, 2013, DOI : 10.2514/6.2013-1512 .

7 Pankonien, A., and Inman, D. J., "Experimental testing of spanwise morphing trailing edge concept," Proceedings of SPIE - The International Society for Optical Engineering, 2013, DOI : 10.1117/12.2009400 .

-8 Bilgen, O., Kochersberger, K. B., Inman, D. J., and Ohanian III, O. J., "Novel, bidirectional, variable-camber airfoil via macro-fiber composite actuators," Journal of Aircraft, vol. 47, 2010, pp. 303-314, DOI : 10.2514/1.45452 .

$\checkmark$ Pankonien, A. M., Duraisamy, K., Faria, C. T., and Inman, D. J., "Synergistic smart morphing aileron: Aerostructural performance analysis," 22nd AIAA/ASME/AHS Adaptive Structures Conference, 2014, DOI : 10.2514/6.2014-0924 .

10 Pankonien, A. M., Faria, C. T., and Inman, D. J., "Synergistic smart morphing aileron: Experimental quasi-static performance characterization," Journal of Intelligent Material Systems and Structures, 2014, DOI : $10.1177 / 1045389$ X14538530 .

-11 Aranakz, A. C., Lakshminarayan, V. K., and Duraisamy, K., "Assessment of transition model and CFD methodology for wind turbine flows," 42nd AIAA Fluid Dynamics Conference and Exhibit 2012, 2012.

12 Pankonien, A. M., and Inman, D. J., "Aeroelastic performance evaluation of a flexure box morphing airfoil concept," Proceedings of SPIE - The International Society for Optical Engineering, 2014, DOI : $10.1117 / 12.2046406$.

13 Ziegler, J. G., and Nichols, N. B., "Optimum settings for automatic controllers," Trans. ASME, vol. 65, 1943, pp. 433-444. 
This article has been cited by:

1. Lawren L. Gamble, Amin Moosavian, Daniel J. Inman. Effects of Speed on Coupled Sweep and Camber in Morphing Wings . [Citation] [PDF] [PDF Plus] 